\title{
Mechanism of resistance to immune checkpoint inhibitors
}

\author{
Manu R. Pandey, Marc S. Ernstoff \\ Department of Medicine, Roswell Park Comprehensive Cancer Center, NY 14203, USA.
}

Correspondence to: Dr. Manu R. Pandey, Department of Medicine, Roswell Park Comprehensive Cancer Center, 665 Elm St, Buffalo, NY 14203, USA. E-mail: manu.pandey@roswellpark.org

How to cite this article: Pandey MR, Ernstoff MS. Mechanism of resistance to immune checkpoint inhibitors. Cancer Drug Resist 2019;2:178-88. http://dx.doi.org/10.20517/cdr.2018.015

Received: 23 Dec 2018 First Decision: 22 Mar 2019 Revised: 16 Apr 2019 Accepted: 8 May 2019 Published: 19 Jun 2019

Science Editor: Helen M. Coley Copy Editor: Cai-Hong Wang Production Editor: Huan-Liang Wu

\begin{abstract}
Immune checkpoint inhibitors $(\mathrm{ICl})$ have revolutionized the management of cancer over the last decade. Instead of targeting the cancer cell directly these agents work by augmenting the immune response towards tumor. Although they are associated with improved responses compared to traditional treatments in several malignancies, a majority of the patients don't respond to ICls even when used in the frontline setting. In patients who do respond, a significant number eventually develop resistance. We will review $\mathrm{ICl}$ mechanisms of action and resistance. We will also discuss new therapeutic options and combinations with other agents that are currently being evaluated to overcome resistance to ICl.
\end{abstract}

Keywords: Immune checkpoint inhibitors, checkpoint inhibitors, resistance, mechanism

\section{INTRODUCTION}

Several immune checkpoint inhibitors (ICI) have been approved for use in metastatic cancers as well as in the adjuvant setting. Ipilimumab, an anti-cytotoxic T-lymphocyte associated protein-4 (CTLA-4) monoclonal antibody (mab) was the first ICI to be approved for use in metastatic melanoma. This was followed by anti-programmed cell death-1 (PD-1) mabs pembrolizumab and nivolumab that were initially approved for melanoma as well, but have since also received approval for several other cancers. The latest ICI to receive approval are anti-programmed death-ligand1 (PD-L1) mabs which include avelumab, druvalumab and atezolizumab.

Although immune checkpoint inhibitors have brought a paradigm shift in the management of several

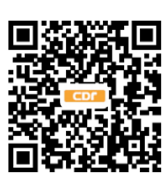


cancers, many patients will have primary resistance to treatment. Ipilimumab, an anti-CTLA4 monoclonal antibody can elicit an objective response in $10 \%-15 \%$ of metastatic melanoma patients ${ }^{[1,2]}$. Even in responders, $40 \%$ of the patients had progression within 2 years ${ }^{[1]}$. PD-1 and PD-L1 inhibitors have an objective response rate of around $20 \%-40 \%$ in a variety of metastatic tumor types ${ }^{[3-6]}$, and like CTLA-4, a significant number of patients lose response to antiPD-1 mabs ${ }^{[7,8]}$. Combinations of anti-CTLA4 + antiPD1 ICIs have also been approved by the FDA demonstrating improved response rates compared to ICI monotherapy, but are associated with significant increases in autoimmune adverse effects ${ }^{[9]}$.

\section{WHAT ARE IMMUNE CHECKPOINTS?}

Immune checkpoints are cellular synapses that are part of the immune system and under physiological condition prevent autoimmunity and regulate immune systems response to infection. Immune checkpoint dysregulation occurs in cancer as a mechanism that leads to tumor escape from immune surveillance. Activation of $\mathrm{T}$ cells requires the interaction of T-cell receptor (TCR) with the complex that includes the major histocompatibility complex (MHC) I or II and cognate antigen present on antigen presenting cells (APC). Engagement of TCR initiates a second interaction between CD28 (on T-cell) with B7 present (on APC), which signals through phosphoinositide-3-Kinase (PI3K) and protein kinase B (AKT) pathway ${ }^{[10]}$. CTLA-4 which is upregulated when T-cells are activated, competitively inhibits the interaction of CD28 with B7 inhibiting T- cell proliferation ${ }^{[11]}$. Downstream inhibition of CD28 mediated PI3K/AKT pathway is another mechanism of $\mathrm{T}$ cell suppression ${ }^{[12]}$. CTLA-4 can also inhibit $\mathrm{T}$ cell activation by trans-endocytosis of B-7 ligands on the APC and degradation in the CTLA-4 expressing cells ${ }^{[13]}$. In addition, CTLA-4 is essential for the suppressive function of $\mathrm{CD} 4 \mathrm{~T}$ regulatory (Treg) cells that is partly mediated by the downregulation of $\mathrm{B} 7$ on $\mathrm{APC}^{[14]}$.

PD-1 is another checkpoint that is expressed upon T cell activation and is present on tumor infiltrating lymphocytes (TIL). Expression of PD-1 in TIL has been shown to be a marker of T-cell exhaustion with decreased production of effector cytokines ${ }^{[15]}$. PD-L1 is expressed on many immune cells as well as on several human malignancies and is induced when cells are exposed to interferon (IFN)- $\gamma^{[16]}$. PD-1/PD-L1 interaction can directly inhibit the TCR signaling by dephosphorylation of downstream molecules ${ }^{[17]}$. Like CTLA-4, PD-1 can inhibit CD28 mediated PI3K/AKT pathway signaling ${ }^{[12]}$. PD-1/PD-L1 pathway also regulates Treg development and functioning mediated by the reduced signaling via the AKT-mTOR pathway; a pathway required for the development of Treg cells ${ }^{[18]}$. Tables 1 and 2 summarize the mechanism of resistance to ICI and potential combinations therapies to overcome ICI resistance.

\section{MECHANISMS OF RESISTANCE: QUALITATIVE AND QUANTITATIVE ASPECTS OF MUTATIONAL BURDEN AND RESPONSES}

Immune recognition of tumor cells requires tumor expression of non-self-antigens (neo-antigens) or embryonic antigens which can be recognized as foreign by the immune system. These neo-antigens are typically derived from a somatic mutation of existing proteins or from the expression of viral proteins that have been incorporated into the cell DNA as part of carcinogenesis ${ }^{[19]}$. Increased tumor somatic mutation burden (TMB) is common in melanoma, non-small lung cancers and bladder cancer ${ }^{[20,21]}$. Although not exclusive, high TMB has been associated with higher ICI response rates and better clinical outcomes in these tumors ${ }^{[2,23]}$. On the other hand, tumors with low TMB like pancreatic cancer ${ }^{[20]}$ have been associated with an immune desert in their tumor microenvironment (TME) and have shown primary resistance to ICI therapy ${ }^{[24]}$. Several strategies have been attempted with varying degrees of success to switch the TME from an immune desert to a site which promotes immune cell infiltration by either inducing mutations and immunogenic cell death using radiation, chemotherapy or oncolytic viruses ${ }^{[25-27]}$.

Tumor heterogeneity and branched evolutionary drift may also contribute to resistance. While tumor heterogeneity has been well recognized in histopathologic specimens, genetic evolution of tumors is now well 
Table 1. Mechanism of primary and acquired resistance to checkpoint inhibitor ${ }^{[99]}$

\begin{tabular}{ll}
\hline Primary resistance & \\
\hline Tumor cell & Low mutation burden/ Lack of neo-antigen \\
& Lower MHC-I expression \\
& Defects in $\beta 2 \mathrm{M}$ \\
& T-cell exclusion program \\
& Overexpression of VEGF \\
& Activation of PI3K-AKT and WNT/ $\beta$-catenin pathways \\
& Mutations in the IFN- $\gamma$ pathway. \\
& Epigenetic modulation of antigen processing and cytokine production \\
& Severe Exhaustion of T-cells \\
& Increase in number and activity of MDSC in the tumor microenvironment \\
& Increase in Tumor associated macrophage \\
& Production of metabolic inhibitors like IDO-1 and adenosine in the tumor microenvironment \\
& Immunosuppressive cytokines like TGF- $\beta$, IL-10 \\
& Defects in $\beta 2 \mathrm{M}$ \\
Acquired resistance & JAK1/2 mutation \\
& Presence of alternative checkpoints \\
\hline
\end{tabular}

MHC: major histocompatibility complex; VEGF: vascular endothelial growth factor; PI3K: phosphoinositide-3-Kinase; AKT: protein kinase B; IFN: interferon; MDSCs: Myeloid-derived suppressor cells; IDO: indoleamine-2,3 dioxygenases; TGF: tumor growth factor; IL: interleukin

documented $^{[28]}$. McGranahan et al. ${ }^{[29]}$ have shown that the presence of intra-tumor heterogeneity defined by the presence of subclones, is associated with lower response rates. They proposed that the presence of several subclones could dampen the $\mathrm{T}$ cell response due to inadequate antigen dosage leading to a less than optimal response to immunotherapy. Tumor heterogeneity has been recognized as a mechanism of drug resistance in chemotherapy and the concepts of how best to sequence or combine therapies can be applied to ICI by recognizing the mechanisms of resistance and opportunities to overcome these pathways ${ }^{[30]}$.

Even though the mutational burden has been shown to be an important aspect of tumor immunogenicity and response to ICI, not all patients with high TMB respond to ICI, and conversely some low TMB patients will respond to therapy. Snyder et al. ${ }^{[23]}$ have shown that although high TMB was associated with longer overall survival with anti-CTLA4 therapy; by itself, TMB was not sufficient to predict clinical response. Rather they proposed the presence of a certain tetrapeptide sequence that is recognized by T-cells as a basis of response to anti-CTLA4 therapy. Hugo et al ${ }^{[31]}$ have shown that response rates to anti-PD1 in melanomas don't correspond to the mutational burden, although a statistically significant relation was seen between high tumor burden and overall survival. Alternatively, this group demonstrated that a gene signature related to mesenchymal transition, wound healing and angiogenesis was associated with tumors unresponsive to antiPD1 therapy and worse overall survival. This signature was upregulated in other tumors including a majority of pancreatic adenocarcinomas which are resistant to anti-PD1 based therapy. Similarly, Jerby-Arnon et al. ${ }^{[32]}$ have defined a resistance program that predicts $\mathrm{T}$ cell exclusion and predicts resistance to immunotherapy. This program was present prior to ICI therapy and enhanced in resistant lesions. The CDK4/6 inhibitor, abemaciclib decreased the expression of this program in melanoma cell lines and showed synergistic action with ICI in murine models. Similar results were also seen in other murine models where the combination of abemaciclib with anti-PD1 not only led to dramatic upregulation of genes related to $\mathrm{T}$ cell activation but also suppression of cell-cycle related genes ${ }^{[33]}$.

T-cell engagement of target requires recognition of the cognate antigen in the context of MHC class I molecules. Lower expression of MHC-I was seen in patients with squamous cell lung cancer and no survival advantage was seen even in patients with high tumor burden ${ }^{[29]}$. Similarly, loss of $\beta 2 \mathrm{M}$, which stabilizes the MHC class I complex, has also been noticed in melanoma treated with dendritic cell vaccine and could be a possible mechanism of resistance ${ }^{[34]}$. Interferons are known to increase expression of class I MHC, $\beta 2 \mathrm{M}$ as well as restore deficiencies in antigen processing pathways [Transporter associated with antigen processing 
Table 2. Combination therapy to overcome resistance to checkpoint inhibitor

\begin{tabular}{|c|c|}
\hline Mechanism of resistance & Potential combination therapy \\
\hline Low mutation burden/lack of neo-antigens & $\begin{array}{l}\mathrm{ICI}+\text { radiation } \\
\mathrm{ICI}+\text { chemotherapy } \\
\mathrm{ICI}+\text { oncolytic virus }\end{array}$ \\
\hline T-Cell Exclusion program & $\mathrm{ICI}+\mathrm{CDK} 4 / 6$ inhibitor \\
\hline Defects in antigen processing $/ \beta 2 \mathrm{M}$ & $\mathrm{ICl}+$ interferon \\
\hline VEGF production by tumor & ICI + VEGF inhibitor \\
\hline Epigenetic modulation leading to decrease in tumor antigenicity & $\begin{array}{l}\mathrm{ICl}+\text { hypo-methylating agent } \\
\mathrm{ICI}+\mathrm{HDAC} \text { inhibitors }\end{array}$ \\
\hline Secondary checkpoints & $\begin{array}{l}\mathrm{ICl}+\text { anti-TIM3 } \\
\mathrm{ICI}+\text { anti-TIGIT }\end{array}$ \\
\hline Immunosuppressive cells in tumor microenvironment & $\begin{array}{l}\mathrm{ICl}+\mathrm{PI} 3 \mathrm{~K}-\gamma \text { selective inhibitor } \\
\mathrm{ICl}+\mathrm{HDAC} \text { inhibitor } \\
\mathrm{ICI}+\text { anti-CSF1R }\end{array}$ \\
\hline Inhibitory molecules and cytokines & $\begin{array}{l}I C I+I D O-1 \text { inhibitor } \\
I C I+\text { anti-CD73mab } \\
I C I+\text { TGF- } \beta \text { receptor kinase }\end{array}$ \\
\hline
\end{tabular}

ICI: Immune checkpoint inhibitors; VEGF: vascular endothelial growth factor; HDAC: histone deacetylase; TGF: tumor growth factor

(TAP) molecules] and have recently been tested in combination with ICIs. Loss of mutations that are associated with neo-antigen formation has also been seen during ICI therapy although the TMB essentially remained unchanged in these patients ${ }^{[35]}$.

\section{LACK OF T-CELL INFILTRATION AND SUPPRESSION OF T CELL FUNCTION}

Immature dendritic cells (DCs) are involved in phagocytosis of tumor antigen and the processing of tumor antigens. They then mature and migrate to secondary lymphoid organs where they interact with naïve $\mathrm{T}$ cell leading to their activation, expansion and later infiltration in the tumor.

Vascular endothelial growth factor (VEGF) is a pro-angiogenic cytokine which is secreted by several solid tumors. It has immunomodulatory actions and has been shown to decrease T-cell proliferation and cytotoxic function ${ }^{[36]}$. Along with this VEGF has been shown to decrease the maturation of DCs ${ }^{[37]}$. Blocking VEGF using monoclonal antibody increased $\mathrm{T}$ cell infiltration into the tumor ${ }^{[38]}$. Supporting the role of VEGF inhibitors in ICI resistance is a phase I study of ipilimumab and bevacizumab in patients with metastatic melanoma which showed a disease control rate of $67 \%$ and median survival of 25 months, which are appreciably more than historical data with ipilimumab monotherapy ${ }^{[39]}$. Downstream pathways of VEGF receptor have also been implicated in resistance formation. Peng et al. ${ }^{[40]}$ have shown that mutated PTEN is associated with inferior response to ICI. PTEN mutated tumors were associated with a decrease in T cell infiltration. PTEN mutation leads to activation of the PI3K-AKT pathway. Combining PI3K inhibitor with ICI showed synergistic anti-tumor activity. The group was also able to show an increase in VEGF production as a possible mechanism, and enhanced $\mathrm{T}$ cell infiltration and antitumor activity when combined with anti-VEGF-blocking antibody. One case report of a patient with metastatic uterine leiomyosarcoma who developed a single ICI resistant metastasis had biallelic PTEN mutation and increased expression of VEGFA which supports this resistance pathway ${ }^{[41]}$.

The activation of the WNT/ $\beta$-catenin pathway has also been shown to be involved with immune evasion. Wnt5a production from melanoma cells also seem to upregulate the $\beta$-catenin pathway in the DCs in the tumor microenvironment leading to differentiation of naive $\mathrm{T}$ cell to Treg cell ${ }^{[42]}$. Activation of the pathway also reduces infiltration and activation of CD103+ DCs due to reduced expression of CCL4 leading to decrease in $\mathrm{T}$ cell infiltration ${ }^{[43]}$. Intra-tumoral injection of dendritic cell restored the responsiveness of tumor to $\mathrm{ICI}^{[43]}$. 
Other important pathways for resistance include STK11/LKB1 mutation in KRAS mutant lung adenocarcinoma that has been shown to directly induce resistance to ICI therapy, with a significant decrease in the response rate and overall survival ${ }^{[4]}$. Another resistance pathway is acquired changes in JAK-STAT. Zaretsky et al. ${ }^{[4]}$ have shown that mutation in Janus Kinase-2 (JAK-2) is a mechanism of resistance to anti-PD1 therapy. They showed that mutation of JAK-2 in melanoma cells leads to a lack of response to IFN- $\gamma$ which includes lack of phosphorylation of STAT-1 and STAT-3 and decreased expression of MHC-1, PD-L1 and TAP-1. Significantly high frequency of mutations in the IFN- $\gamma$ pathway was also seen in patients who were non-responders to ipilimumab compared to patients who responded to therapy ${ }^{[46]}$.

Epigenetic modulation has also been implicated in resistance to ICI. Epigenetic modulation has been shown to decrease the expression of tumor-associated antigen through alteration of the antigen processing machinery, downregulation of MHC-I and the decreased expression of MHC-I on the cell surface ${ }^{[47]}$. Treatment of renal cancer cells with DNA hypomethylating agent induces the expression of cancer testes antigens, which are normally not expressed by these cells ${ }^{[48]}$. In melanoma cell lines these agents increase the expression of MHC-I and increase gp100 specific CTL mediated lysis ${ }^{[49]}$. Similarly, when mice and human melanoma cell lines were treated with histone deacetylase inhibitor panobinostat, an increase in the expression of MHC-I along with other costimulatory molecules was seen ${ }^{[50]}$. Increased expression of melanoma differentiation antigens was also seen in these cell lines ${ }^{[50]}$. Peng et al. ${ }^{[51]}$ have also shown in mouse ovarian cell line models that DNA methylation decreases the production of tumor-mediated Th1 type cytokines including CXCL9 and CXCL10, leading to decreased T cell infiltration into the tumor. Treatment with epigenetic modulator was associated increase in the expression of these cytokines and had synergistic activity in tumor control when combined with T-cells. Several other clinical trials combining epigenetic modifiers and ICI are ongoing ${ }^{[52]}$. A phase 2 study of the combination of nivolumab with azacitidine (a hypomethylating agent) in patients with relapsed/refractory acute myeloid leukemia showed an overall response rate(ORR) of 33\% which was higher than historical ORR with azacitidine alone ${ }^{[53]}$.

\section{PRESENCE OF ALTERNATIVE CHECKPOINTS AND SEVERE T CELL EXHAUSTION}

Several other checkpoints apart for PD-1 and CTLA-4 have been discovered which contribute to tumor resistance to available ICI therapy. T-cell immunoglobulin mucin (TIM)-3 is found on Th1 and cytotoxic T cell and act as a negative regulator of their function ${ }^{[54]}$. It also forms a part of the exhausted $\mathrm{T}$ cell phenotype which is associated with a lack of proliferation and cytotoxic activity ${ }^{[54]}$. Like TIM-3, lymphocyte activating gene (LAG)-3 is another checkpoint which is expressed on exhausted T cells ${ }^{[55]}$. B- and T-lymphocyte attenuator (BLTA) is a checkpoint that has been shown to be a late marker of exhaustion. CD8+BLTA+ $\mathrm{T}$ cells showed high co-expression of other inhibitory receptors compared to CD8+ cells with other inhibitory receptors ${ }^{[56]}$. Koyama et al.$^{[57]}$ showed an increase in the surface levels of TIM-3 and LAG-3 on CD8 cells in murine models of lung cancer resistant to anti-PD1 therapy when compared to untreated tumors. Addition of antibody to TIM-3 after the development of anti-PD1 resistance lead to an increase in overall survival of mice. An increase in overall survival was seen when anti-PD1 was added to antiTIM-3 therapy in murine models of glioblastoma ${ }^{[58]}$. Similarly, T-cell immunoglobulin and ITIM domain (TIGIT) is another checkpoint which is expressed on CD8+ T-cells, NK cells and Treg ${ }^{[59]}$. In-vitro PD-1 blockade causes upregulation of TIGIT in tumor antigen-specific CD8+ T-cells, a potential mechanism of resistance. Dual blockade of TIGIT and PD-1 increased IFN- $\gamma$, TNF and T cell proliferation ${ }^{[59]}$. V-domain Ig suppressor of T-cell activation (VISTA) belongs to the immunoglobulin superfamily and is another immune checkpoint with potent negative regulation of T-cell function. VISTA is expressed at high levels on monocytes, granulocytes, and macrophages, and at lower densities on T-cell populations within the tumor microenvironment. Kuklinski et al ${ }^{[60]}$ found that expression of VISTA in primary melanomas was associated with a significantly worse disease-specific survival, and is regulated differently than PD1 and represents another potential pathway of resistance to established ICIs. 
Blackburn et al. ${ }^{[61]}$ reported two subsets of CD8+ cells in mice chronically infected with lymphocytic choriomeningitis virus based on expression of PD-1. The cells with intermediate PD-1 expression were rescuable with blockade of PD-1/PD-L1 pathway with more expansion and protective immunity, and reduced apoptosis when compared to cells with high PD-1 expression which were not rescued by using anti-PD-L1. CD8+ T cells with high PD-1 expression (PD-1 high) also express other inhibitory checkpoints associated with $\mathrm{T}$ cell exhaustion and the expression of multiple inhibitory checkpoints correlates inversely with $\mathrm{T}$ cell function as evident by lower staining for intracellular IFN- $\gamma$ and $\mathrm{TNF}^{[62]}$. Different stages of $\mathrm{T}$ cell exhaustion have been described, starting with the loss of production of IL-2 followed by TNF and ultimately $\mathrm{IFN}^{[63]}$. Presence of partially exhausted CD8+ T cells capable of producing IFN but not IL-2 or TNF correlated with response to anti-PD1 therapy in patients with melanoma ${ }^{[64]}$. The development of an exhausted and dysfunctional state has been linked to epigenetic modulation in genes involved in effector function and memory $\mathrm{T}$ cell differentiation ${ }^{[65,66]}$.

\section{IMMUNOSUPPRESSIVE TUMOR MICROENVIRONMENT}

Myeloid-derived suppressor cells (MDSCs) alter the function of CD8+ T cells ${ }^{[67]}$. Several mechanisms have been proposed including a decrease in arginine and cysteine, production of free radicals which eventually inhibit the functioning of TCR and IL-2 signaling, decreased trafficking of T cells into the lymph node and tumor, induction of $\mathrm{T}$ cell apoptosis and expansion of Tregs ${ }^{[67]}$. Presence of higher baseline numbers of MDSCs in peripheral blood has been seen in non-responders when compared with responders and was also associated with worse overall survival in patients with metastatic melanoma who were on ipilimumab ${ }^{[68]}$. In addition in non-responders, the immunosuppressive activity of MDSCs has been shown to be significantly higher when compared to responders while getting treatment with ipilimumab ${ }^{[69]}$. Similarly, in patients treated with nivolumab after progression on ipilimumab higher baseline MDSC number correlated with progression and poor overall survival ${ }^{[70]}$. Highfil et al ${ }^{[71]}$ have shown in a murine model of rhabdomyosarcoma that CXCL-1 and CXCL-2 are responsible for the trafficking of MDSCs into the tumor. There was a significant tumor shrinkage in the tumor with the use of anti-PD1 in mice reconstituted with CXCR-2 (-/-) hematopoietic cells when compared to wild type mice. De Henau et al ${ }^{[72]}$ showed that inhibition of PI3K- $\gamma$ decreased myeloid cell migration into the tumor. Combination of ICI and PI3K- $\gamma$ selective inhibitor significantly delayed tumor growth in previously ICI resistant tumors. Combination of HDAC inhibitor entinostat and ICI has also been shown to have synergistic effects, capable of sensitizing tumors which are resistant to ICI. This appears to be mediated by a decrease in the immunosuppressive potential of $\operatorname{MDSC}^{[73]}$. Blood and tissue MDSCs also correlate with several clinicopathologic factors and may predict for pathological complete response providing further evidence that MDSCs play an important role across tumor types ${ }^{[74]}$.

Tumor-associated macrophages (TAM) are associated with poor survival in several malignancies ${ }^{[75-77]}$. DeNardo et al ${ }^{[77]}$ showed an inverse relation between infiltration of CD68+ macrophages and CD8+ T-cells in a breast cancer model. They also proposed that colony stimulating factor(CSF)- 1 is responsible for macrophage infiltration, and its blockade is responsible for the decrease in TAM, increase in CD8+ cells tumor infiltration, increase in expression of cytotoxic effector molecules and decrease in the expression of arginase-1. Similar results have been seen in other tumor models too ${ }^{[78,79]}$. CFS- $1 /$ CSF-1R signal blockade modestly reduces $\mathrm{T}$ cell growth when used as a single agent due to upregulation of $\mathrm{T}$ cell checkpoint. Combination of ICI and CSF1R blockade, on the other hand showed impressive tumor control in murine models of pancreatic carcinoma, which is generally resistant to ICI $^{[80]}$.

Indoleamine-2,3 dioxygenases (IDO) is an IFN- $\gamma$ induced intracellular enzyme which is involved in tryptophan metabolism ${ }^{[81]}$. It is expressed by several human tumor cell lines and activated DCs and is shown to decrease $\mathrm{T}$ cell proliferation ${ }^{[81-83]}$. IDO causes inhibition of $\mathrm{T}$ cell proliferation by decreasing tryptophan which is an essential amino acid and production of metabolites which have immunosuppressive activity ${ }^{[81,84]}$. 
Holmgaard et al ${ }^{[85]}$ showed that in murine models of melanoma combination of anti-CTLA4 with antiIDO resulted in a significant decrease in tumor sizes and improved overall survival. Monotherapy with either agent didn't have any significant effects. Similarly, in a murine model of glioblastoma combination of anti-PD-L1, anti-CTLA, and IDO inhibitor lead to a durable response in all animals ${ }^{[86]}$. Unfortunately, the phase III study of pembrolizumab $+/$ - epacadostat did not find any benefit from the addition of the IDO1 inhibitor $^{[87]}$. On the other hand, an earlier study of an IDO1 inhibitor with ipilimumab in patients with metastatic melanoma has been reported that showed good tolerance and disease control rate of $75 \%{ }^{[88]}$, but will need to be followed by a well-designed phase III study.

Adenosine which is hydrolyzed from AMP by the extracellular domain of CD73 present on tumor cells has also been implicated in apoptosis of $\mathrm{T}$ cells and suppression of their activation and effector function ${ }^{[89]}$. The combination of anti-PD1 or anti-CTLA-4 mab with anti-CD73mab has been shown to have impressive therapeutic activity and increases survival in a murine model. This was associated with an increase in antigen-specific CD8+ TILs, increase in Th1 related genes, and a decrease in expression of CD73 on tumor and TILs ${ }^{[90]}$. Tumor growth factor (TGF) $\beta$ is another cytokine that is produced by the tumor and the microenvironment. In normal tissues, it prevents tumorigenesis and maintains homeostasis although in cancerous tissue it promotes tumor progression and escape from immune surveillance ${ }^{[91]}$. Thymoma murine model which secretes TGF- $\beta$ has been shown to decrease the cytotoxic activity of CD $8+\mathrm{T}$ cells ${ }^{[92]}$. Combination of anti-CTLA- 4 with TGF- $\beta$ receptor kinase inhibitor in melanoma murine model showed synergistic activity with an increase in the CD $8+/$ Treg ratio ${ }^{[93]}$. Phase I trials of TGF- $\beta$ inhibitors as monotherapy and in combination with ICI are currently ongoing ${ }^{[94]}$. Immune suppressive factors in the tumor microenvironment TME have also been suggested as pathways of resistance. Tumor-derived immunosuppressive cytokines, like IL-10 and exosomes, have been noted ${ }^{[95,96]}$. These observations will lead to novel combinatorial therapies directed at these pathways.

Prior treatment may also influence changes in tumor microenvironment causing resistance to ICI. Hugo et al ${ }^{[97]}$ have shown that in melanoma which develops resistance to MAPKi there is positive enrichment in genes related to inflammation (likely mediated by macrophages) and monocyte. There was a decrease in CD8 $\mathrm{T}$ cells (overall and melanoma-tumor reactive) along with downregulation of genes involved in antigen processing and presentation. This was corroborated in a retrospective study which showed that in patients who discontinued BRAFi with/without MEKi, there were no objective responses with ipilimumab ${ }^{[98]}$.

\section{CONCLUSION}

Although ICIs have had a major impact on the therapy of many tumor types and subsequent patients' lives, the observation of primary and acquired resistance to anti-PD1/PD-L1 and anti-CTLA4 agents leave much room for improvement. Understanding the biology of tumor-associated immune responses and the multiple pathways of T cell inhibition and exhaustion will provide opportunities to enhance the therapeutic window.

\section{DECLARATIONS}

\section{Authors' contributions}

Both authors contributed equally to the work.

\section{Availability of data and materials}

Not applicable.

\section{Financial support and sponsorship}

None. 


\section{Conflicts of interest}

Both authors declared that there are no conflicts of interest.

\section{Ethical approval and consent to participate}

Not applicable.

\section{Consent for publication}

Not applicable.

\section{Copyright}

(c) The Author(s) 2019.

\section{REFERENCES}

1. Hodi FS, O’Day SJ, McDermott DF, Weber RW, Sosman JA, et al. Improved survival with ipilimumab in patients with metastatic melanoma. N Engl J Med 2010;363:711-23.

2. Robert C, Thomas L, Bondarenko I, O’Day S, Weber J, et al. Ipilimumab plus dacarbazine for previously untreated metastatic melanoma. N Engl J Med 2011;364:2517-26.

3. Robert C, Schachter J, Long GV, Arance A, Grob JJ, et al. Pembrolizumab versus Ipilimumab in Advanced Melanoma. N Engl J Med 2015;372:2521-32.

4. Robert C, Long GV, Brady B, Dutriaux C, Maio M, et al. Nivolumab in previously untreated melanoma without BRAF mutation. N Engl J Med 2015;372:320-30.

5. Borghaei H, Paz-Ares L, Horn L, Spigel DR, Steins M, et al. Nivolumab versus Docetaxel in Advanced Nonsquamous Non-Small-Cell Lung Cancer. N Engl J Med 2015;373:1627-39.

6. Powles T, Duran I, van der Heijden MS, Loriot Y, Vogelzang NJ, et al. Atezolizumab versus chemotherapy in patients with platinumtreated locally advanced or metastatic urothelial carcinoma (IMvigor211): a multicentre, open-label, phase 3 randomised controlled trial. Lancet 2018;391:748-57.

7. Gettinger SN, Horn L, Gandhi L, Spigel DR, Antonia SJ, et al. Overall survival and long-term safety of nivolumab (anti-programmed death 1 antibody, BMS-936558, ONO-4538) in patients with previously treated advanced non-small-cell lung cancer. J Clin Oncol 2015;33:2004-12.

8. Schachter J, Ribas A, Long GV, Arance A, Grob JJ, et al. Pembrolizumab versus ipilimumab for advanced melanoma: final overall survival results of a multicentre, randomised, open-label phase 3 study (KEYNOTE-006). Lancet 2017;390:1853-62.

9. Larkin J, Chiarion-Sileni V, Gonzalez R, Grob JJ, Cowey CL, et al. Combined nivolumab and ipilimumab or monotherapy in untreated melanoma. N Engl J Med 2015;373:23-34.

10. Miller J, Baker C, Cook K, Graf B, Sanchez-Lockhart M, et al. Two pathways of costimulation through CD28. Immunol Res 2009;45:159-72.

11. Walunas TL, Lenschow DJ, Bakker CY, Linsley PS, Freeman GJ, et al. CTLA-4 can function as a negative regulator of T cell activation. Immunity 1994;1:405-13.

12. Parry RV, Chemnitz JM, Frauwirth KA, Lanfranco AR, Braunstein I, et al. CTLA-4 and PD-1 receptors inhibit T-cell activation by distinct mechanisms. Mol Cell Biol 2005;25:9543-53.

13. Qureshi OS, Zheng Y, Nakamura K, Attridge K, Manzotti C, et al. Trans-endocytosis of CD80 and CD86: a molecular basis for the cellextrinsic function of CTLA-4. Science 2011;332:600-3.

14. Wing K, Onishi Y, Prieto-Martin P, Yamaguchi T, Miyara M, et al. CTLA-4 control over Foxp3+ regulatory T cell function. Science 2008;322:271-5.

15. Ahmadzadeh M, Johnson LA, Heemskerk B, Wunderlich JR, Dudley ME, et al. Tumor antigen-specific CD8 T cells infiltrating the tumor express high levels of PD-1 and are functionally impaired. Blood 2009;114:1537-44.

16. Dong H, Strome SE, Salomao DR, Tamura H, Hirano F, et al. Tumor-associated B7-H1 promotes T-cell apoptosis: a potential mechanism of immune evasion. Nat Med 2002;8:793-800.

17. Yokosuka T, Takamatsu M, Kobayashi-Imanishi W, Hashimoto-Tane A, Azuma M, et al. Programmed cell death 1 forms negative costimulatory microclusters that directly inhibit T cell receptor signaling by recruiting phosphatase SHP2. J Exp Med 2012;209:120117.

18. Francisco LM, Salinas VH, Brown KE, Vanguri VK, Freeman GJ, et al. PD-L1 regulates the development, maintenance, and function of induced regulatory T cells. J Exp Med 2009;206:3015-29.

19. Schumacher TN, Schreiber RD. Neoantigens in cancer immunotherapy. Science 2015;348:69-74.

20. Lawrence MS, Stojanov P, Mermel CH, Robinson JT, Garraway LA, et al. Discovery and saturation analysis of cancer genes across 21 tumour types. Nature 2014;505:495-501.

21. Alexandrov LB, Nik-Zainal S, Wedge DC, Aparicio SA, Behjati S, et al. Signatures of mutational processes in human cancer. Nature 2013;500:415-21. 
22. Rizvi NA, Hellmann MD, Snyder A, Kvistborg P, Makarov V, et al. Cancer immunology. Mutational landscape determines sensitivity to PD-1 blockade in non-small cell lung cancer. Science 2015;348:124-8.

23. Snyder A, Makarov V, Merghoub T, Yuan J, Zaretsky JM, et al. Genetic basis for clinical response to CTLA-4 blockade in melanoma. N Engl J Med 2014;371:2189-99.

24. Royal RE, Levy C, Turner K, Mathur A, Hughes M, et al. Phase 2 trial of single agent Ipilimumab (anti-CTLA-4) for locally advanced or metastatic pancreatic adenocarcinoma. J Immunother 2010;33:828-33.

25. Deng L, Liang H, Burnette B, Beckett M, Darga T, et al. Irradiation and anti-PD-L1 treatment synergistically promote antitumor immunity in mice. J Clin Invest 2014;124:687-95.

26. Menard C, Martin F, Apetoh L, Bouyer F, Ghiringhelli F. Cancer chemotherapy: not only a direct cytotoxic effect, but also an adjuvant for antitumor immunity. Cancer Immunol Immunother 2008;57:1579-87.

27. Ribas A, Dummer R, Puzanov I, VanderWalde A, Andtbacka RHI, et al. Oncolytic Virotherapy Promotes Intratumoral T Cell Infiltration and Improves Anti-PD-1 Immunotherapy. Cell 2017;170:1109-19 e10.

28. Gerlinger M, Rowan AJ, Horswell S, Math M, Larkin J, et al. Intratumor heterogeneity and branched evolution revealed by multiregion sequencing. N Engl J Med 2012;366:883-92.

29. McGranahan N, Furness AJ, Rosenthal R, Ramskov S, Lyngaa R, et al. Clonal neoantigens elicit T cell immunoreactivity and sensitivity to immune checkpoint blockade. Science 2016;351:1463-9.

30. Day RS. Treatment sequencing, asymmetry, and uncertainty: protocol strategies for combination chemotherapy. Cancer Res 1986;46:3876-85.

31. Hugo W, Zaretsky JM, Sun L, Song C, Moreno BH, et al. Genomic and Transcriptomic Features of Response to Anti-PD-1 Therapy in Metastatic Melanoma. Cell 2017;168:542.

32. Jerby-Arnon L, Shah P, Cuoco MS, Rodman C, Su MJ, et al. A Cancer Cell Program Promotes T Cell Exclusion and Resistance to Checkpoint Blockade. Cell 2018;175:984-97.e24.

33. Schaer DA, Beckmann RP, Dempsey JA, Huber L, Forest A, et al. The CDK4/6 Inhibitor Abemaciclib Induces a T Cell Inflamed Tumor Microenvironment and Enhances the Efficacy of PD-L1 Checkpoint Blockade. Cell Rep 2018;22:2978-94.

34. del Campo AB, Kyte JA, Carretero J, Zinchencko S, Mendez R, et al. Immune escape of cancer cells with beta2-microglobulin loss over the course of metastatic melanoma. Int J Cancer 2014;134:102-13.

35. Anagnostou V, Smith KN, Forde PM, Niknafs N, Bhattacharya R, et al. Evolution of Neoantigen Landscape during Immune Checkpoint Blockade in Non-Small Cell Lung Cancer. Cancer Discov 2017;7:264-76.

36. Mulligan JK, Day TA, Gillespie MB, Rosenzweig SA, Young MR. Secretion of vascular endothelial growth factor by oral squamous cell carcinoma cells skews endothelial cells to suppress T-cell functions. Hum Immunol 2009;70:375-82.

37. Oyama T, Ran S, Ishida T, Nadaf S, Kerr L, et al. Vascular endothelial growth factor affects dendritic cell maturation through the inhibition of nuclear factor-kappa B activation in hemopoietic progenitor cells. J Immunol 1998;160:1224-32.

38. Shrimali RK, Yu Z, Theoret MR, Chinnasamy D, Restifo NP, et al. Antiangiogenic agents can increase lymphocyte infiltration into tumor and enhance the effectiveness of adoptive immunotherapy of cancer. Cancer Res 2010;70:6171-80.

39. Hodi FS, Lawrence D, Lezcano C, Wu X, Zhou J, et al. Bevacizumab plus ipilimumab in patients with metastatic melanoma. Cancer Immunol Res 2014;2:632-42.

40. Peng W, Chen JQ, Liu C, Malu S, Creasy C, et al. Loss of PTEN Promotes Resistance to T Cell-Mediated Immunotherapy. Cancer Discov 2016;6:202-16.

41. George S, Miao D, Demetri GD, Adeegbe D, Rodig SJ, et al. Loss of PTEN Is Associated with Resistance to Anti-PD-1 Checkpoint Blockade Therapy in Metastatic Uterine Leiomyosarcoma. Immunity 2017;46:197-204.

42. Holtzhausen A, Zhao F, Evans KS, Tsutsui M, Orabona C, et al. Melanoma-Derived Wnt5a Promotes Local Dendritic-Cell Expression of IDO and Immunotolerance: Opportunities for Pharmacologic Enhancement of Immunotherapy. Cancer Immunol Res 2015;3:1082-95.

43. Spranger S, Bao R, Gajewski TF. Melanoma-intrinsic beta-catenin signalling prevents anti-tumour immunity. Nature 2015;523:231-5.

44. Skoulidis F, Goldberg ME, Greenawalt DM, Hellmann MD, Awad MM, et al. STK11/LKB1 Mutations and PD-1 Inhibitor Resistance in KRAS-Mutant Lung Adenocarcinoma. Cancer Discov 2018;8:822-35.

45. Zaretsky JM, Garcia-Diaz A, Shin DS, Escuin-Ordinas H, Hugo W, et al. Mutations Associated with Acquired Resistance to PD-1 Blockade in Melanoma. N Engl J Med 2016;375:819-29.

46. Gao J, Shi LZ, Zhao H, Chen J, Xiong L, et al. Loss of IFN-gamma Pathway Genes in Tumor Cells as a Mechanism of Resistance to Anti-CTLA-4 Therapy. Cell 2016;167:397-404 e9.

47. Sigalotti L, Fratta E, Coral S, Maio M. Epigenetic drugs as immunomodulators for combination therapies in solid tumors. Pharmacol Ther 2014;142:339-50.

48. Coral S, Sigalotti L, Altomonte M, Engelsberg A, Colizzi F, et al. 5-aza-2'-deoxycytidine-induced expression of functional cancer testis antigens in human renal cell carcinoma: immunotherapeutic implications. Clin Cancer Res 2002;8:2690-5.

49. Fonsatti E, Nicolay HJ, Sigalotti L, Calabro L, Pezzani L, et al. Functional up-regulation of human leukocyte antigen class I antigens expression by 5-aza-2'-deoxycytidine in cutaneous melanoma: immunotherapeutic implications. Clin Cancer Res 2007;13:3333-8.

50. Woods DM, Woan K, Cheng F, Wang H, Perez-Villarroel P, et al. The antimelanoma activity of the histone deacetylase inhibitor panobinostat (LBH589) is mediated by direct tumor cytotoxicity and increased tumor immunogenicity. Melanoma Res 2013;23:341-8.

51. Peng D, Kryczek I, Nagarsheth N, Zhao L, Wei S, et al. Epigenetic silencing of TH1-type chemokines shapes tumour immunity and immunotherapy. Nature 2015;527:249-53.

52. Aspeslagh S, Morel D, Soria JC, Postel-Vinay S. Epigenetic modifiers as new immunomodulatory therapies in solid tumours. Ann Oncol 2018;29:812-24. 
53. Daver N, Garcia-Manero G, Basu S, Boddu PC, Alfayez M, et al. Efficacy, Safety, and Biomarkers of Response to Azacitidine and Nivolumab in Relapsed/Refractory Acute Myeloid Leukemia: A Non-randomized, Open-label, Phase 2 Study. Cancer Discov 2018; doi: 10.1158/2159-8290.CD-18-0774.

54. Zhu C, Anderson AC, Kuchroo VK. TIM-3 and its regulatory role in immune responses. Curr Top Microbiol Immunol 2011;350:1-15.

55. Wherry EJ, Ha SJ, Kaech SM, Haining WN, Sarkar S, et al. Molecular signature of CD8+ T cell exhaustion during chronic viral infection. Immunity 2007;27:670-84.

56. Thommen DS, Schreiner J, Muller P, Herzig P, Roller A, et al. Progression of Lung Cancer Is Associated with Increased Dysfunction of T Cells Defined by Coexpression of Multiple Inhibitory Receptors. Cancer Immunol Res 2015;3:1344-55.

57. Koyama S, Akbay EA, Li YY, Herter-Sprie GS, Buczkowski KA, et al. Adaptive resistance to therapeutic PD-1 blockade is associated with upregulation of alternative immune checkpoints. Nat Commun 2016;7:10501.

58. Kim JE, Patel MA, Mangraviti A, Kim ES, Theodros D, et al. Combination Therapy with Anti-PD-1, Anti-TIM-3, and Focal Radiation Results in Regression of Murine Gliomas. Clinical Cancer Research 2017;23:124-36.

59. Chauvin JM, Pagliano O, Fourcade J, Sun Z, Wang H, et al. TIGIT and PD-1 impair tumor antigen-specific CD8(+) T cells in melanoma patients. J Clin Invest 2015;125:2046-58.

60. Kuklinski LF, Yan S, Li Z, Fisher JL, Cheng C, et al. VISTA expression on tumor-infiltrating inflammatory cells in primary cutaneous melanoma correlates with poor disease-specific survival. Cancer Immunol Immunother 2018;67:1113-21.

61. Blackburn SD, Shin H, Freeman GJ, Wherry EJ. Selective expansion of a subset of exhausted CD8 T cells by alphaPD-L1 blockade. Proc Natl Acad Sci U S A 2008;105:15016-21.

62. Blackburn SD, Shin H, Haining WN, Zou T, Workman CJ, et al. Coregulation of CD8+ T cell exhaustion by multiple inhibitory receptors during chronic viral infection. Nat Immunol 2009;10:29-37.

63. Wherry EJ, Blattman JN, Murali-Krishna K, van der Most R, Ahmed R. Viral persistence alters CD8 T-cell immunodominance and tissue distribution and results in distinct stages of functional impairment. J Virol 2003;77:4911-27.

64. Daud AI, Loo K, Pauli ML, Sanchez-Rodriguez R, Sandoval PM, et al. Tumor immune profiling predicts response to anti-PD-1 therapy in human melanoma. J Clin Invest 2016;126:3447-52.

65. Schietinger A, Philip M, Krisnawan VE, Chiu EY, Delrow JJ, et al. Tumor-Specific T Cell Dysfunction Is a Dynamic Antigen-Driven Differentiation Program Initiated Early during Tumorigenesis. Immunity 2016;45:389-401.

66. Philip M, Fairchild L, Sun L, Horste EL, Camara S, et al. Chromatin states define tumour-specific T cell dysfunction and reprogramming. Nature 2017;545:452-6.

67. Gabrilovich DI, Ostrand-Rosenberg S, Bronte V. Coordinated regulation of myeloid cells by tumours. Nat Rev Immunol 2012;12:253-68.

68. Sade-Feldman M, Kanterman J, Klieger Y, Ish-Shalom E, Olga M, et al. Clinical Significance of Circulating CD33+CD11b+HLA-DRMyeloid Cells in Patients with Stage IV Melanoma Treated with Ipilimumab. Clin Cancer Res 2016;22:5661-72.

69. Gebhardt C, Sevko A, Jiang H, Lichtenberger R, Reith M, et al. Myeloid Cells and Related Chronic Inflammatory Factors as Novel Predictive Markers in Melanoma Treatment with Ipilimumab. Clin Cancer Res 2015;21:5453-9.

70. Weber J, Gibney G, Kudchadkar R, Yu B, Cheng P, et al. Phase I/II Study of Metastatic Melanoma Patients Treated with Nivolumab Who Had Progressed after Ipilimumab. Cancer Immunol Res 2016;4:345-53.

71. Highfill SL, Cui Y, Giles AJ, Smith JP, Zhang H, et al. Disruption of CXCR2-mediated MDSC tumor trafficking enhances anti-PD1 efficacy. Sci Transl Med 2014;6:237ra67.

72. De Henau O, Rausch M, Winkler D, Campesato LF, Liu C, et al. Overcoming resistance to checkpoint blockade therapy by targeting PI3Kgamma in myeloid cells. Nature 2016;539:443-7.

73. Christmas BJ, Rafie CI, Hopkins AC, Scott BA, Ma HS, et al. Entinostat Converts Immune-Resistant Breast and Pancreatic Cancers into Checkpoint-Responsive Tumors by Reprogramming Tumor-Infiltrating MDSCs. Cancer Immunol Res 2018;6:1561-77.

74. Ornstein MC, Diaz-Montero CM, Rayman P, Elson P, Haywood S, et al. Myeloid-derived suppressors cells (MDSC) correlate with clinicopathologic factors and pathologic complete response $(\mathrm{pCR})$ in patients with urothelial carcinoma (UC) undergoing cystectomy. Urol Oncol 2018;36:405-12.

75. Steidl C, Lee T, Shah SP, Farinha P, Han G, et al. Tumor-associated macrophages and survival in classic Hodgkin's lymphoma. N Engl J Med 2010;362:875-85.

76. Kurahara H, Shinchi H, Mataki Y, Maemura K, Noma H, et al. Significance of M2-polarized tumor-associated macrophage in pancreatic cancer. J Surg Res 2011;167:e211-9.

77. DeNardo DG, Brennan DJ, Rexhepaj E, Ruffell B, Shiao SL, et al. Leukocyte complexity predicts breast cancer survival and functionally regulates response to chemotherapy. Cancer Discov 2011;1:54-67.

78. Strachan DC, Ruffell B, Oei Y, Bissell MJ, Coussens LM, et al. CSF1R inhibition delays cervical and mammary tumor growth in murine models by attenuating the turnover of tumor-associated macrophages and enhancing infiltration by CD8 $(+) \mathrm{T}$ cells. Oncoimmunology 2013;2:e26968.

79. Ries CH, Cannarile MA, Hoves S, Benz J, Wartha K, et al. Targeting tumor-associated macrophages with anti-CSF-1R antibody reveals a strategy for cancer therapy. Cancer Cell 2014;25:846-59.

80. Zhu Y, Knolhoff BL, Meyer MA, Nywening TM, West BL, et al. CSF1/CSF1R blockade reprograms tumor-infiltrating macrophages and improves response to T-cell checkpoint immunotherapy in pancreatic cancer models. Cancer Res 2014;74:5057-69.

81. van Baren N, Van den Eynde BJ. Tryptophan-degrading enzymes in tumoral immune resistance. Front Immunol 2015;6:34.

82. Hwu P, Du MX, Lapointe R, Do M, Taylor MW, et al. Indoleamine 2,3-dioxygenase production by human dendritic cells results in the inhibition of T cell proliferation. J Immunol 2000;164:3596-9. 
83. Uyttenhove C, Pilotte L, Theate I, Stroobant V, Colau D, et al. Evidence for a tumoral immune resistance mechanism based on tryptophan degradation by indoleamine 2,3-dioxygenase. Nat Med 2003;9:1269-74.

84. Frumento G, Rotondo R, Tonetti M, Damonte G, Benatti U, et al. Tryptophan-derived catabolites are responsible for inhibition of T and natural killer cell proliferation induced by indoleamine 2,3-dioxygenase. J Exp Med 2002;196:459-68.

85. Holmgaard RB, Zamarin D, Munn DH, Wolchok JD, Allison JP. Indoleamine 2,3-dioxygenase is a critical resistance mechanism in antitumor T cell immunotherapy targeting CTLA-4. J Exp Med 2013;210:1389-402.

86. Wainwright DA, Chang AL, Dey M, Balyasnikova IV, Kim CK, et al. Durable therapeutic efficacy utilizing combinatorial blockade against IDO, CTLA-4, and PD-L1 in mice with brain tumors. Clin Cancer Res 2014;20:5290-301.

87. Long GV, Dummer R, Hamid O, Gajewski T, Caglevic C, et al. Epacadostat (E) plus pembrolizumab (P) versus pembrolizumab alone in patients (pts) with unresectable or metastatic melanoma: results of the phase 3 ECHO-301/KEYNOTE-252 study. J Clin Oncol 2018;36:108.

88. Gibney GT, Hamid O, Gangadhar TC, Lutzky J, Olszanski AJ, et al. Preliminary results from a phase $1 / 2$ study of INCB024360 combined with ipilimumab (ipi) in patients (pts) with melanoma. J Clin Oncol 2014;32:3010.

89. Jin D, Fan J, Wang L, Thompson LF, Liu A, et al. CD73 on tumor cells impairs antitumor T-cell responses: a novel mechanism of tumor-induced immune suppression. Cancer Res 2010;70:2245-55.

90. Allard B, Pommey S, Smyth MJ, Stagg J. Targeting CD73 enhances the antitumor activity of anti-PD-1 and anti-CTLA-4 mAbs. Clin Cancer Res 2013; 19:5626-35.

91. Massague J. TGFbeta in Cancer. Cell 2008;134:215-30.

92. Thomas DA, Massague J. TGF-beta directly targets cytotoxic T cell functions during tumor evasion of immune surveillance. Cancer Cell 2005;8:369-80.

93. Hanks BA, Holtzhausen A, Evans K, Heid M, Blobe GC. Combinatorial TGF- $\beta$ signaling blockade and anti-CTLA-4 antibody immunotherapy in a murine BRAFV600E-PTEN-/- transgenic model of melanoma. J Clin Oncol 2014;32:3011.

94. de Gramont A, Faivre S, Raymond E. Novel TGF-beta inhibitors ready for prime time in onco-immunology. Oncoimmunology 2017;6:e1257453.

95. La Shu S, Yang Y, Allen CL, Maguire O, Minderman H, et al. Metabolic reprogramming of stromal fibroblasts by melanoma exosome microRNA favours a pre-metastatic microenvironment. Sci Rep 2018;8:12905.

96. Tuccitto A, Tazzari M, Beretta V, Rini F, Miranda C, et al. Immunomodulatory factors control the fate of melanoma tumor initiating cells. Stem Cells 2016;34:2449-60.

97. Hugo W, Shi H, Sun L, Piva M, Song C, et al. Non-genomic and immune evolution of melanoma acquiring MAPKi resistance. Cell 2015; $162: 1271-85$.

98. Ackerman A, Klein O, McDermott DF, Wang W, Ibrahim N, et al. Outcomes of patients with metastatic melanoma treated with immunotherapy prior to or after BRAF inhibitors. Cancer 2014;120:1695-701.

99. Sharma P, Hu-Lieskovan S, Wargo JA, Ribas A. Primary, adaptive, and acquired resistance to cancer immunotherapy. Cell 2017;168:707-23. 